\title{
Comparaison des performances de l'oie, du canard mulard et du canard de Barbarie soumis au gavage
}

\author{
G Guy, D Rousselot-Pailley, D Gourichon
}

INRA, station expérimentale des palmipèdes à foie gras, Artiguères, F40280 Benquet, France

(Reçu le 10 juin 1993 ; accepté le 4 october 1994)

\begin{abstract}
Résumé - Cent vingt palmipèdes répartis en 3 lots sont élevés à la station expérimentale des palmipèdes à foie gras d'Artiguères à raison de : 40 oisons de type Landais mâles et femelles, 40 canards mulards mâles, 40 canards de Barbarie mâles. Ces animaux subissent un programme d'alimentation propre à chaque type de palmipède et destiné à optimiser leur préparation en vue d'une mise en gavage. En revanche, de nombreux facteurs (choix du maïs, des locaux de gavage, matériel, opérateur...) sont harmonisés. Au cours du gavage d'une durée identique de $13 \mathrm{j}$, tous les palmipèdes engraissent de façon spectaculaire $(+41 \%$ de gain de poids pour les canards de Barbarie et respectivement +53 et $+54 \%$ pour les oisons et pour les canards mulards). Les quantités de foie gras produites sont différentes, les foies d'oies sont les plus lourds $(793 \mathrm{~g})$, les foies de canards mulards sont intermédiaires $(702 \mathrm{~g})$, les foies de canard de Barbarie sont les plus légers $(560 \mathrm{~g})$. La qualité technologique des foies exprimée par l'aptitude à retenir les graisses pendant la cuisson classe les animaux dans le même ordre : le foie gras d'oie présente la meilleure qualité. Pour la production de viande, c'est le canard de Barbarie qui présente le meilleur rendement (les muscles des cuisses et des pectoraux représentent $17,3 \%$ du poids vif contre $15 \%$ et $13 \%$ respectivement chez le canard mulard et chez l'oie). Les oisons sont les moins performants, mais ils sont placés en gavage à un âge $(13,5$ semaines $)$ qui ne correspond pas à la pleine maturité. Finalement, en fonction des objectifs poursuivis, tous les types de palmipèdes peuvent être choisis, mais nous montrons pourquoi le canard mulard très rustique et qui présente toujours des niveaux de production proches des meilleurs est le palmipède le plus employé en gavage.
\end{abstract}

élevage / gavage / oie / canard mulard / canard de Barbarie / foie gras

Summary - Comparison of geese, mule duck and muscovy duck after cramming. 120 waterfowl of 3 types were raised at the station expérimentale des palmipèdes à foie gras in Artiguères as follows: 40 Landaise geese, 20 males and 20 females, 40 mule ducks, males only, 40 muscovy ducks, males. The feeding program was specific to each type of bird. The purpose of such an approach was to adapt birds in the best way before cramming. However, numerous parameters (choice of corn, cramming room, material and operator) have been standardized. Cramming lasted for $13 \mathrm{~d}$. At the end of this period, each type of bird presented a spectacular fattening, ranging from $+41 \%$ for muscovy ducks to $+53 \%$ for geese and $+54 \%$ for mule ducks. Furthermore, "foie gras" production was also 
very different between each type of waterfowl since it ranged from 560 to $793 \mathrm{~g}$ for the muscovy ducks and geese, respectively. The performance of mule ducks was intermediate $(702 \mathrm{~g})$ and significantly different from the other waterfowl. The technical quality of "foie gras", appreciated by the amount of fat loss during thermic processing, showed the same classification: $21.2 \%$ for geese, $43.9 \%$ for mule ducks and $55.6 \%$ for muscovy ducks. Muscovy ducks present the best yield for breast muscles; geese are the least productive for this parameter, but they present the best yield for thigh muscles. It appears that starting the cramming treatment at 13 weeks for geese does not correspond to the total maturity of these birds. Depending on the purpose of each one, all these waterfowl can be used, but it seems that there is by now an overall advantage to using mule ducks for cramming.

breeding / cramming / geese / mule ducks / muscovy ducks / foie gras

\section{INTRODUCTION}

En France, la production de foie gras fait appel à 3 types de palmipèdes : le canard de Barbarie (Cairina moschata) ; le canard mulard : croisement intergénérique d'un canard de Barbarie mâle et d'une cane commune (Anas platyrhynchos); certaines souches d'oies (Anser anser).

L'objet de cette étude concerne l'élevage et la préparation des animaux ainsi que l'étude des principaux rendements zootechniques des 3 types de palmipèdes.

La composition des foies gras de ces animaux a par ailleurs été déterminée après mesures des caractéristiques biochimiques (Salichon et al, 1994).

Le canard mulard est très rustique, ses capacités d'ingestion et son aptitude à engraisser en un temps très court en font le palmipède le plus utilisé en gavage (plus de 12 millions de têtes par an). Après le gavage, l'animal est valorisé par son foie gras mais aussi par les parties carnées qui représentent environ $40 \%$ du prix de vente.

Le canard de Barbarie a un potentiel d'ingestion de maïs en gavage moindre que le canard mulard. La durée du gavage est plus longue d'environ $3 \mathrm{j}$, soit $18 \mathrm{j}$ au lieu de 15 , en moyenne. La valorisation de l'animal gavé se fait pour moitié par le foie gras et pour l'autre moitié par les parties carnées.

Chez les canards, seuls les mâles sont utilisés en gavage, en général à l'âge de 12 sem. Les canes présentent des niveaux de production très inférieurs en qualité et en quantité. Les canettes mulardes sont éliminées à la naissance, les femelles Barbarie sont utilisées comme canettes à rôtir.

L'oie présente le plus fort gabarit des 3 palmipèdes, mais le potentiel d'ingestion instantanée le plus faible, ce qui entraîne des gavages plus ou moins longs ( 18 à $24 \mathrm{~h}$ en moyenne) selon la fréquence des repas. L'âge de mise en gavage est plus élevé, environ $18 \mathrm{sem}$. Les mâles et les femelles sont utilisés compte tenu du faible potentiel de reproduction de cette espèce (25 à 30 oisons pour les souches à foie gras par femelle et par an). De plus, les animaux des 2 sexes ont des performances très voisines. La qualité reconnue du foie gras d'oie explique que cet organe assure la majeure partie des recettes, jusqu'à $75 \%$ de l'animal gavé.

Les résultats technico-économiques des différents palmipèdes soumis au gavage ont déjà été comparés (Khoel, 1993). Compte tenu de la spécialisation des ateliers, les comparaisons n'ont été faites que sur des animaux élevés et gavés dans des conditions très diverses. De ce fait, les différences observées peuvent être imputables à de multiples facteurs : la conduite des animaux en élevage-prégavage ; le choix de la technique de gavage ; le confort de la salle de gavage (ventilation, climatisation, matériel) ; la dextérité du gaveur ; le choix du maïs. 
Le but de cette étude est de comparer les différents types d'animaux, dans des conditions fixées et contrôlées pour chaque type. Toutefois, la conduite alimentaire en élevage comporte des similitudes, soit 3 phases distinctes : alimentation à volonté (pour assurer le démarrage et la croissance du jeune palmipède) ; rationnement dont l'objectif est double : économie d'aliment et entraînement à une alimentation par repas ; phase de prégavage pendant laquelle on augmente chaque jour la ration jusqu'à obtenir des ingérés bien supérieurs à une alimentation ad libitum sans rationnement préalable.

Cette dernière phase a pour effets : un début de stéatose hépatique comme l'a constaté Babilé (1989) ; une distention de l'œsophage du palmipède ; une préparation de l'animal à ingérer une quantité importante d'aliment dans un temps très court, situation qu'il va rencontrer au cours du gavage.

La phase de prégavage est très importante, et son succès conditionne la réussite du gavage.

Lors de cette étude, même si les paimipèdes n'ont pas suivi exactement le même schéma d'élevage pour optimiser leurs performances en gavage, leur préparation a été semblable. En outre, nous avons imposé des conditions de gavage identiques : salle de gavage, gaveuse, maïs, opérateur.

\section{MATÉRIEL ET MÉTHODES}

Quarante animaux de chaque type de palmipède sont mis en élevage à la station d'Artiguères à l'âge de $1 \mathrm{j}$, soit :

- 40 canards de Barbarie mâles ST4 - société France-Canard,

- 40 canards mulards mâles, fils de canes Aliénor-SICA SEPALM,

-40 oisons, 20 mâles et 20 femelles, de souche Landaise OO "souche foie" (INRA, Artiguères).

Tous les animaux sont démarrés sur litière de copeaux et passent sur caillebotis intégral à l'âge de 4 sem. Ils reçoivent en élevage un aliment commercial (société Maïsadour) dont les teneurs en matières protéiques brutes (MPB) et les niveaux énergétiques sont les suivants :

Canards : 1 à $7 \mathrm{j}$, aliment démarrage, 20,5\% MPB*, $2920 \mathrm{kcal} / \mathrm{kg} ; 8$ à $28 \mathrm{j}$, aliment croissance, $18,2 \% \mathrm{MPB}^{\star}, 2750 \mathrm{kcal} / \mathrm{kg} ; 28$ à $84 \mathrm{j}$, aliment finition, 15,5\% MPB*, $2750 \mathrm{kcal} / \mathrm{kg}$.

Oisons : 1 à $35 \mathrm{j}$, aliment démarrage, $15,8 \%$ $\mathrm{MPB}^{\star}, 2850 \mathrm{kcal} / \mathrm{kg} ; 36$ à $56 \mathrm{j}$, aliment croissance, $15,0 \% \mathrm{MPB}, 2850 \mathrm{kcal} / \mathrm{kg} ; 57$ à $93 \mathrm{j}$, aliment finition, $13,8 \% \mathrm{MPB}^{*}, 2600 \mathrm{kcal} / \mathrm{kg}$; avec $\mathrm{MPB}=$ matière protéique brute $(\mathrm{N} \times 6,25)$.

On notera que l'aliment commercial proposé pour l'élevage des oisons présente des caractéristiques assez basses, notamment au niveau protéique, par rapport aux recommandations faites par Leclercq et Rousselot-Pailley (1982). Après une courte période d'alimentation où ils sont nourris ad libitum (4 sem pour les oisons et 6 sem pour les canetons), les animaux subissent un rationnement dont le niveau est adapté à chaque type de palmipède. La phase de prégavage d'une durée de $4 \mathrm{j}$ pour les canards de Barbarie et de $10 \mathrm{j}$ pour les oisons et les canards mulards termine la période d'élevage. Pendant cette phase, on utilise le comportement alimentaire généré par le rationnement pour augmenter chaque jour le niveau des rations et entraîner l'animal vers des consommations spontanées très élevées.

Les animaux sont ensuite répartis en salle de gavage à une température de $16^{\circ} \mathrm{C}$ et une hygrométrie de $80 \%$ destinées à leur assurer un confort optimum dont l'influence est importante sur les résultats. Les palmipèdes sont placés dans des épinettes collectives (type Pujol) à raison de 4 canards ou 3 oies par épinette. Le choix de ce matériel se justifie par le fait qu'il permet de gaver indifféremment les oies ou les canards et qu'il limite la pénibilité du gavage, le parcours des palmipèdes étant restreint.

Tous les animaux subissent un gavage très intensif d'une durée identique de $13 \mathrm{j}$. Avec 2 repas par jour, la durée du gavage chez les canards est plutôt courte par rapport aux durées constatées sur le terrain. Le gavage des oies est exceptionnellement rapide, une technique de gavage mise au point à la station d'Artiguères avec 6 interventions par jour permet d'obtenir ce résultat. L'âge de mise en gavage est de $12 \mathrm{sem}$ pour les canards et de $13,5 \mathrm{sem}$ pour les oies. La distribution du maïs cuit est effectuée par un seul opérateur avec une gaveuse à vis sans fin de 
type Mirandaise. Pour chaque type de palmipède, nous pratiquons un gavage "au toucher" où le gaveur décide de la quantité à distribuer à chaque oiseau.

Après $13 \mathrm{j}$ de gavage, tous les animaux sont abattus, après une durée de jeûne identique de $12 \mathrm{~h}$. Une éviscération à chaud est pratiquée, les foies gras sont pesés et refroidis rapidement sur lit de glace, ce qui améliore leur qualité technologique (Castaing et Robin, 1990 ; Rousselot- Pailley et al, 1992). Le lendemain, ces foies subissent une mesure de qualimétrie (mesure d'impédance électrique qui permet de prédire la perte de graisse ou "fonte lipidique" au cours du chauffage : corrélation voisine de $0,85 \mathrm{chez}$ l'oie (RousselotPailley et al, 1993). Un test de fonte est également réalisé pour tous les foies sur un échantillon de $60 \mathrm{~g}$ autoclavé à $105^{\circ} \mathrm{C}$ pendant $50 \mathrm{~min}$.

Après avoir prélevé et pesé l'ensemble des paletots ( 2 cuisses plus pilons, 2 magrets plus manchons, le croupion), 12 animaux par espèce subissent une dissection anatomique, pour évaluer la production de cuisse et de magret. Des échantillons de muscle (Pectoralis major) et de foie gras sont prélevés pour déterminer leur teneur en lipides : extraction à chaud à l'éther éthylique et extraction à froid au chloroformeméthanol selon la méthode de Folch pour les muscles (Folch et al, 1957). L'extraction à froid prend en compte tous les lipides ; l'éther éthylique n'extrait que les graisses neutres.

Les analyses statistiques sont réalisées en analyse de variance avec la procédure GLM (General linear method) du logiciel SAS (Statistical analysis system), le risque de $1^{\text {re }}$ espèce ayant été fixé au seuil de $5 \%$.

\section{RÉSULTATS}

\section{En élevage (tableau I)}

Mise en place du rationnement :

- oisons, à 29 j ;

- canetons (mulards et de Barbarie), à 43 j.

Lorsqu'on exprime les consommations en cumul d'aliment sur toute la période d'élevage, les canards de Barbarie utilisent 14,4 $\mathrm{kg}$ d'aliment, les canards mulards $17,9 \mathrm{~kg}$ et les oies $22,5 \mathrm{~kg}$, mais ces dernières sont alimentées jusqu'à l'âge de 13,5 sem vs 12 sem pour les canards.

\section{Le poids vif (tableau II)}

Le démarrage des canards mulards est beaucoup plus rapide que celui des canards de Barbarie. Par la suite, ces derniers rattrapent leur retard de croissance et sont les plus lourds à la mise en gavage.

Les oisons perdent du poids entre 56 et $81 \mathrm{j}$, probablement par suite d'un rationnement trop sévère. Ce rationnement est cependant très efficace pour accroître la consommation en fin de prégavage $: 700 \mathrm{~g}$ le dernier jour.

Enfin les âges de pesée des palmipèdes au début du prégavage et à la mise en gavage diffèrent pour les canards gavés à $12 \mathrm{sem}$ et les oies à $13,5 \mathrm{sem}$.

\section{Les indices de consommation (tableau III)}

Durant la phase d'élevage ils sont très nettement en faveur des canards de Barbarie qui ont un indice de 3,24 à la mise en gavage, inférieur à celui des canards mulards $(4,37)$ et à celui des oisons $(4,73)$.

\section{En gavage}

\section{Consommation de maïs (fig 1 )}

Les consommations sont exprimées en "maïs sec» avant la cuisson, ce grain présente tout de même environ $13 \%$ d'humidité.

$\mathrm{Au}$ total les oies ingèrent plus de maïs sec $(11,7 \mathrm{~kg})$ que les canards mulards $(10,7 \mathrm{~kg})$ et les canards de Barbarie $(8,1$ $\mathrm{kg})$. Ces consommations sont les moyennes de 40 animaux par type de palmipède et correspondent aux quantités de maïs que le gaveur juge bon d'administrer à chaque animal. 
Tableau I. Consommation par jour et par animal.

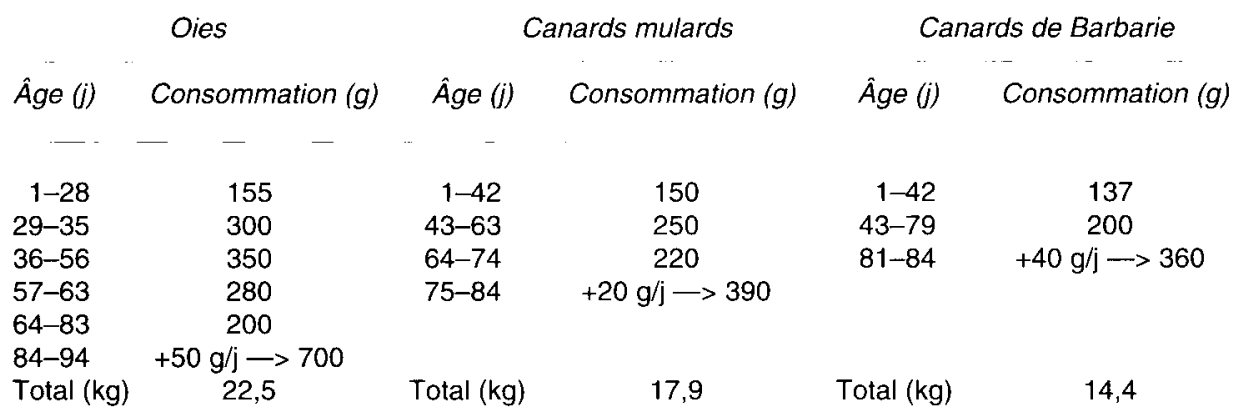

Tableau II. Évolution du poids vif en élevage (en g).

$\begin{array}{cccc}\text { Âge }(j) & \text { Oies } & \text { Canards mulards } & \text { Canards de Barbaie } \\ \ldots & & - & \\ 1 & 93 \pm 8 & 44 \pm 4 & 41 \pm 4 \\ 28 & 1763 \pm 182 & 1852 \pm 138 & 1347 \pm 117 \\ 56 & 4535 \pm 347 & 3588 \pm 257 & 3383 \pm 232 \\ 74 & - & 3929^{\mathrm{a}} \pm 369 & 4156^{\mathrm{a}} \pm 317 \\ 81 & 4152^{\mathrm{a}} \pm 365 & - & - \\ 84 & 4847^{\mathrm{b}} \pm 446 & 4138^{\mathrm{b}} \pm 389 & 4474^{\mathrm{b}} \pm 350 \\ 94 & & - & -\end{array}$

Moyennes \pm écarts types. a poids début de prégavage ; ${ }^{b}$ poids de mise en gavage.

\section{Performances en gavage (tableau IV)}

Les 3 types de palmipède donnent des foies gras de poids significativement différents : respectivement 793,702 et $560 \mathrm{~g}$ pour les oies, les canards mulards et les canards de Barbarie. Le classement des foies après le gavage est le même que celui observé chez les animaux maigres non prégavés (environ 100,55 et $50 \mathrm{~g}$ ).

Le poids de foie gras exprimé par rapport au poids vif de l'animal est sensiblement identique chez les oies $(10,65 \%)$ et chez les canards mulards $(10,98 \%)$ et diffère significativement de celui du canard de Barbarie $(8,90 \%)$.
Tableau III. Évolution des indices de consommation.

$\begin{array}{cccc}\text { Âge (j) } & \text { Oies } & \begin{array}{c}\text { Canards } \\ \text { mulards }\end{array} & \begin{array}{c}\text { Canards } \\ \text { de Barbarie }\end{array} \\ 28 & 1,40 & 1,59 & 1,59 \\ 56 & 2,65 & 2,74 & 2,57 \\ 74 & - & 3,89 & 2,96 \\ 81 & 4,37 & - & - \\ 84 & - & 4,37 & 3,24 \\ 91 & 4,73 & - & -\end{array}$




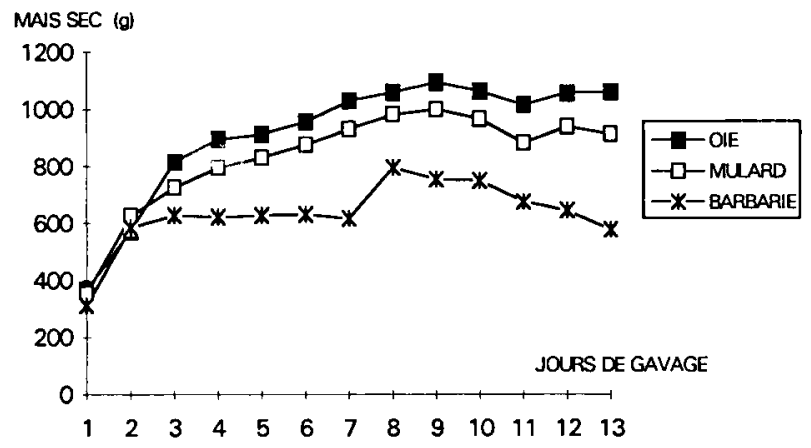

Fig 1. Évolution de la consommation de maïs au cours du gavage.

La quantité de maïs consommé en gavage par $\mathrm{kg}$ de foie gras produit est très similaire entre les différents palmipèdes : respectivement 14,8 pour les oies, 15,2 pour les canards mulards et 14,5 pour les canards de Barbarie.

En revanche, si on prend en compte le foie réellement consommable, c'est l'oie qui est la plus performante du fait de la faible valeur de la fonte lipidique de son foie. Les valeurs de «foie restant» sont de $625 \mathrm{~g}$ pour les oies, de $394 \mathrm{~g}$ pour les canards mulards et de $249 \mathrm{~g}$ pour les canards de Barbarie.

Les mesures de qualimétrie sont un moyen de classer les foies gras à l'intérieur d'un type de palmipède (plus la valeur est élevée, plus la fonte lipidique est impor- tante). Les valeurs moyennes observées par type de palmipèdes classent les foies dans le même ordre de qualité que les tests de fonte.

Le tableau $V$ permet de comparer les performances des oisons en fonction du sexe. Seul le poids vif à la mise en gavage est significativement plus élevé chez les mâles $(5031 \mathrm{~g})$ que chez les femelles (4 $704 \mathrm{~g})$.

Au terme du gavage, bien que l'écart entre les poids vifs soit du même ordre de grandeur (261 g au lieu de $327 \mathrm{~g}$ ), il n'est plus significatif.

La variabilité individuelle et le nombre des animaux testés expliquent que les para-

Tableau IV. Performances en gavage.

\begin{tabular}{|c|c|c|c|c|c|}
\hline & Oies & Canards mulards & Canards de Barbarie & $E^{2} M^{\mathrm{a}}$ & $\mathrm{P}$ \\
\hline - & ... & & & -. & -- \\
\hline Poids départ gavage $(\mathrm{g})$ & $4868^{a}$ & $4133^{c}$ & $4468^{b}$ & 409 & $<0,001$ \\
\hline Poids fin gavage $(\mathrm{g})$ & $7444^{a}$ & $6387^{b}$ & $6314^{b}$ & 580 & $<0,001$ \\
\hline Poids saigné plumé $(\mathrm{g})$ & $6768^{a}$ & $5680^{\mathrm{b}}$ & $5444^{c}$ & 495 & $<0,001$ \\
\hline Paletot $(\mathrm{g})$ & $2935^{a}$ & $2688^{b}$ & $2413^{c}$ & 238 & $<0,001$ \\
\hline Foie $(\mathrm{g})$ & $793^{a}$ & $702^{b}$ & $560^{c}$ & 151 & $<0,001$ \\
\hline Foie (\% poids vif) & $10,65^{a}$ & $10,98^{a}$ & $8,90^{\mathrm{b}}$ & 1,92 & $<0,001$ \\
\hline Qualimétrie & $33,4^{c}$ & $56,4^{b}$ & $88,5^{a}$ & 38,2 & $<0,001$ \\
\hline Fonte lipidique (\%) & $21,2^{\mathrm{c}}$ & $43,9^{b}$ & $55,6^{a}$ & 10,8 & $<0,001$ \\
\hline
\end{tabular}

a Écart type moyen. b,c Différence significative pour les valeurs d'une même ligne suivies d'une lettre différente. 
mètres mesurés (toujours favorables aux mâles) ne puissent être trouvés significativement différents.

\section{Dissections anatomiques (tableaux VI et VII)}

Le poids des cuisses des différents types de palmipède suit le même classement que les poids vifs à l'abattage. Les masses musculaires de la cuisse sont les plus importantes chez l'oie (268 g) que chez les canards de Barbarie ( $228 \mathrm{~g}$ ) et les canards mulards $(204 \mathrm{~g})$. Le poids des os de la cuisse montre que le canard mulard a un squelette très fin comparé à celui du canard de Barbarie et surtout à celui de l'oie.

Le développement du magret est le plus important chez les canards de Barbarie $(420 \mathrm{~g})$ qui présentent pourtant les poids les plus faibles à l'abattage, à l'inverse les oisons qui sont les animaux les plus lourds n'offrent que $351 \mathrm{~g}$ de magret. Lorsqu'on observe la quantité de viande des magrets, les différences entre les types de palmipède sont encore plus accusées.

La viande d'oie est la plus riche en lipides, la viande des canards mulards est intermédiaire et la viande des canards de Barbarie est la plus pauvre, quel que soit le mode d'extraction : éther éthylique à chaud qui n'extrait que les graisses neutres ou le chloroforme-méthanol qui prend également en compte les phospholipides.

\section{DISCUSSION}

Il semble bien que la maîtrise de l'élevage des canards destinés au gavage soit satisfaisante tant chez le canard de Barbarie que chez le canard mulard. En revanche, l'élevage des oisons destinés au gavage présente de nombreuses difficultés car leurs besoins alimentaires sont mal connus. II est nécessaire de les rationner fortement pour générer un comportement boulimique garantissant le succès de prégavage. De plus, l'âge de 13,5 sem à la mise en gavage n'assure pas la maturité des masses musculaires pectorales, l'oison se comportant comme les autres palmipèdes qui développent leurs filets plus tardivement que les membres postérieurs (Richard et al, 1985). Chez l'oison, la croissance s'achève beaucoup plus tard - vers 16 ou 18 sem d'âge (Dubois, 1993). Pendant le gavage, les palmipèdes ne développent guère leurs masses musculaires (Babilé, 1989), les oisons mis en gavage à 13,5 sem n'ont donc

Tableau V. Performances des oisons en fonction du sexe.

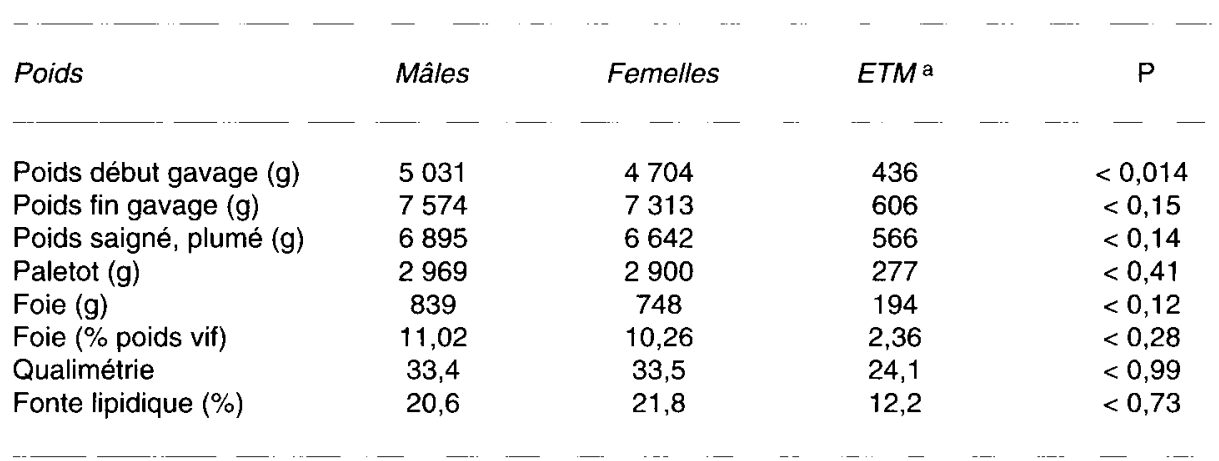

\footnotetext{
a Écart type moyen.
} 
Tableau VI. Poids (en g) des constituants de la carcasse séparés par dissection.

$\begin{array}{lccccc} & \text { Oies } & \text { Canards mulards } & \text { Canards de Barbarie } & E T M & P \\ \text { Cuisse } & & & & - & - \\ \text { Os de la cuisse } & 577^{\mathrm{a}} & 493^{\mathrm{b}} & 441^{\mathrm{c}} & 70 & <0,001 \\ \text { Peau de la cuisse } & 53^{\mathrm{a}} & 32^{\mathrm{c}} & 38^{\mathrm{b}} & 10 & <0,001 \\ \text { Muscle de la cuisse } & 268^{\mathrm{a}} & 254^{\mathrm{a}} & 172^{\mathrm{b}} & 47 & <0,001 \\ \text { Magret } & 204^{\mathrm{c}} & 228^{\mathrm{b}} & 34 & <0,001 \\ \text { Peau du magret } & 351^{\mathrm{b}} & 408^{\mathrm{a}} & 420^{\mathrm{a}} & 51 & <0,001 \\ \text { Muscle du magret } & 136^{\mathrm{a}} & 133^{\mathrm{a}} & 101^{\mathrm{b}} & 25 & <0,001 \\ & 214^{\mathrm{c}} & 273^{\mathrm{b}} & 318^{\mathrm{a}} & 51 & <0,001\end{array}$

Différence significative pour les valeurs d'une même ligne suivie d'une lettre différente.

Tableau VII. Composition en lipides des magrets (en \% de poids sec).

$\begin{array}{ccccc}\text { Oies } & \begin{array}{c}\text { Canards } \\ \text { mulards }\end{array} & \begin{array}{c}\text { Canards } \\ \text { de Barbarie }\end{array} & \text { ETM } & \text { P } \\ 25,6^{a} & 19,4^{\mathrm{b}} & 13,8^{\mathrm{c}} & 5,5 & <0,001 \\ 32,1^{\mathrm{a}} & 26,3^{\mathrm{b}} & 19,4^{\mathrm{c}} & 6,2 & <0,001\end{array}$

Différence significative pour les valeurs d'une même ligne suivie d'une lettre différente.

pas extériorisé tout leur potentiel de production. II n'est pas pour autant acquis qu'il faille retarder l'âge de mise en gavage, cela dépend de l'utilisation que l'on souhaite faire de la viande des oies. En tout état de cause, cela augmenterait un indice de consommation déjà très élevé chez cet animal.

Le gavage d'une durée de 13 j pour tous les palmipèdes est très rapide, les résultats en valeur pondérale sont néanmoins très élevés. Le succès de cette opération est pour une grande part conditionné par la préparation des animaux. En effet, lors de gavage court, la distribution d'une quantité très importante de maîs dès le début n'est possible que si les animaux ont une capacité d'absorption suffisante, conséquence d'un prégavage réussi.
Chez les oisons, les résultats en fonction du sexe montrent que les femelles sont moins performantes que les mâles, mais, compte tenu des faibles effectifs et de la variabilité des caractères, la plupart des paramètres ne diffèrent pas significativement. Le poids de foie présente une différence de $90 \mathrm{~g}$ en faveur des mâles assortie d'une fonte lipidique plus faible de 1,2 point. Ces résultats sont en opposition avec l'habituelle relation entre le poids de foie et la fonte lipidique. Ceci suggère que la production des femelles pourrait être pénalisée en quantité mais aussi en qualité.

Les dissections anatomiques placent l'oie en tête pour le poids des cuisses qui est lié au gabarit de l'animal. Pour le magret, les 2 types de canard sont meilleurs producteurs 
que l'oie pour des raisons d'âge évoquées précédemment.

Le canard de Barbarie présente les muscles du magret les plus lourds probablement parce qu'il a été sélectionné pour un meilleur rendement en viande. Les poids de muscle pectoral relevés dans le tableau VI confirment la maturité des 2 types de canards, les valeurs étant égales ou légèrement supérieures aux observations faites par d'autres auteurs (Babilé, 1989 ; Castaing et Robin, 1990).

\section{CONCLUSION}

Les résultats de cette expérience justifient pleinement l'utilisation massive du canard mulard pour le gavage. II permet des performances qui sont souvent intermédiaires entre les 2 autres types de palmipède. II est, en outre, très rustique et le développement de l'insémination artificielle apporte la maîtrise de la production des canetons. L'engouement pour la viande de canard cuisinée sous diverses formes (magret, confits, ...) ainsi que l'utilisation du foie gras sous des formes différentes (foie frais ou mi-cuit) militent pour l'utilisation accrue de ce palmipède, ainsi que pour celle du canard de Barbarie.

Dans un marché du foie gras en pleine expansion, le nombre d'oies mises en gavage est stable ou même en régression. Les causes en sont principalement les difficultés d'approvisionnement en oisons, la pénibilité du gavage, les difficultés d'adaptation de l'espèce aux systèmes de production très intensifs, les coûts de production élevés insuffisamment compensés par les prix de vente. Quoi qu'il en soit, ce palmipède trouvera toujours des adeptes, certains gourmets ne jurant que par le foie d'oie qui est, par ailleurs, le seul à pouvoir supporter des traitements thermiques très poussés nécessités par certaines conserves.

\section{RÉFÉRENCES}

Babilé R (1989) La production de foies gras de canard de Barbarie (Cairina moschata) : aspects génétiques, nutritionnels et technologiques. Thèse doct Ėtat, INP Toulouse, $n^{\circ 156,315 p}$

Castaing J, Robin N (1990) Alimentation énergétique du canard mulard. Rev Alim Anim 440, 22-25

Dubois JP (1993) L'alimentation des oies à gaver. tres Journées de la recherche sur les palmipèdes à foie gras, Bordeaux, 35-48

Folch J, Less M. Sloane-Stanley GH (1957) A simple method for the isolation and purification of total lipids from animal tissues. J Biol Chem 226, 497-509

Khoel PF (1993) Les résultats technico-économiques des paimipèdes à foie gras. fres journées de la recherche sur les palmipèdes à foie gras, Bordeaux, 165-180

Leclercq B, Rousselot Pailley D (1982) L'alimentation protéique de l'oison à gaver. Archiv Geflügelkunde $46,167-170$

Ricard FH, de Carville H, Marche G (1985) Étude comparative de la composition anatomique des canards de Barbarie, Pékin et mulard. Les colloques de I'INRA 42, 75-99

Rousselot-Pailley D, Guy G, Gourichon D, Sellier N, Blum JC (1992) Influence des conditions d'abattage et de réfrigération sur la qualité des foies gras d'oie. INRA Prod Anim 5, 167-172

Rousselot-Pailley D, Rouvier R, Bartel G (1993) Méthodologie du test de fonte des foies gras, fres Journées de recherche sur les palmipèdes à foie gras, Bordeaux, 73-86

Salichon MR, Guy G, Rousselot-Pailley D, Blum JC (1994) Composition des 3 types de foie gras : oie, canard mulard et canard de Barbarie. Ann Zootech $43,213-220$ 